\title{
Kostenerstattungsanspruch des Käufers nach eigenmächtiger Selbstvornahme der Mängelbeseitigung?
}

Von Dr. Ulrich G. Schroeter, Berlin

\section{Problemstellung}

Ein Recht des Käufers, die Mängel einer gelieferten Sache ohne vorherige Rücksprache mit dem Verkäufer selbst zu beseitigen und diesem die dabei angefallenen Kosten sodann (ganz oder zum Teil) in Rechnung zu stellen, ist im modernisierten Schuldrecht des BGB nicht ausdrücklich vorgesehen. Lorenz hat gleichwohl vor kurzem die Ansicht vertreten, dass ein solches Käuferrecht besteht ${ }^{1}$. Im Anschluss an eine ablehnende Erwiderung im Schrifttum $^{2}$ hat sich daraus zwischenzeitlich ein wissenschaftlicher Disput zum neuen Kaufrecht entwickelt, der mit einiger Vehemenz ausgetragen wird ${ }^{3}$. Nachdem sich nunmehr erstmals auch zwei Instanzgerichte explizit zur Frage eines Kostenerstattungsanspruches des Käufers nach eigenmächtiger Selbstvornahme der Mängelbeseitigung geäußert haben ${ }^{4}$, sollen die einschlägigen Argumente nochmals kritisch hinterfragt und ergänzt werden.

\section{1. "Eigenmächtige Selbstvornahme« der Mängel- beseitigung durch den Käufer}

Die hier interessierenden Fälle der Selbstvornahme - also der vom Käufer vorgenommenen Beseitigung eines Sachmangels, mit dem die verkaufte Sache zum Zeitpunkt des Gefahrenüberganges ( $\$ 434$ Abs. 1 S. 1 BGB) behaftet war - lassen sich dabei dann als »eigenmächtig« qualifizieren, wenn der Käufer dem Verkäufer zuvor keine (in den $\$ \$ 281$ Abs. 1 Satz 1, 323 Abs. 1 BGB vorausgesetzte) angemessene Frist zur Nacherfüllung gesetzt hat, eine solche nicht gemäß den $\$ \$ 281$ Abs. 2, 323 Abs. 2, 440 BGB ausnahmsweise entbehrlich war $^{5}$ und der Käufer die Mängelbeseitigung auch nicht im Auftrag des Verkäufers durchführt.

\section{2. §326 Abs. 2 S. 2 BGB (analog) als Grundlage für einen Kostenerstattungsanspruch?}

In den beschriebenen Fällen der eigenmächtigen Selbstvornahme soll dem Käufer nach von Lorenz vertretener Auffassung gleichwohl ein Erstattungsanspruch in Höhe der vom Verkäufer ersparten Nachbesserungsaufwendungen zustehen; selbiger sei auf $\$ 326$ Abs. 2 S. 2 BGB in direkter oder entsprechender Anwendung zu stützen und auf den Kaufpreisanspruch des Verkäufers anzurechnen ${ }^{6}$. Begründet wird dies mit dem Gedanken, dass ab dem Zeitpunkt, zu dem der Käufer den Mangel selbst (etwa durch Reparatur) beseitigt hat, rechtlich kein behebbarer, sondern ein unbehebbarer Mangel vorliege; die Mängelbeseitigung im Wege der Nacherfüllung sei damit unmöglich geworden. Die Folge sei zum einen, dass die Pflicht des Käufers zur Kaufpreiszahlung gemäß $\$ 326$ Abs. 1 S. 2 BGB zunächst ungemindert erhalten bleibe. Sie werde erst durch einen Rücktritt oder eine Minderungserklärung des Käufers berührt, welche jedoch daran scheiterten, dass $\$ 326$ Abs. 5 BGB auch $₫ 323$ Abs. 6 BGB in Bezug nehme und diese Vorschrift einen Rücktritt dann (vollständig) ausschließe, wenn der Gläubiger für den Umstand, der ihn zum
Rücktritt berechtigt (also die nunmehrige Mängelfreiheit nach Reparatur) »allein oder weit überwiegend verantwortlich ist «dies sei in Fällen der Selbstvornahme durch den Käufer der Fall, da dieser die Unmöglichkeit der Nacherfüllung ersichtlich allein zu verantworten habe. Wegen $\$ 441$ Abs. 1 S. 1 BGB (»Statt zurückzutreten ...«) entfalle damit auch das Minderungsrecht, und mangels Vertretenmüssens des Verkäufers scheide auch ein Anspruch auf Schadensersatz statt der Leistung ( $\$ \$ 280$ Abs. 1, 3 i. V. m. 283 BGB) aus 7.

Gleichwohl verbleibe ein »Nachgeschmack « aufgrund der Tatsache, dass der Verkäufer nun die Aufwendungen erspare, die er im Falle der Nacherfüllung gemäß $\$ 439$ Abs. 2 BGB gehabt hätte. Es liege insoweit eine »evident unbeabsichtigte«, planwidrige Regelungslücke des Unmöglichkeitsrechts vor: Da es sich bei der Lieferung unbehebbar mangelhafter Sachen konstruktiv um einen Fall teilweiser Unmöglichkeit (in Bezug auf die Qualität) handele, in welchem gemäß $\$ 326$ Abs. 2 S. 2 BGB der Anspruch auf die Gegenleistung nur unter Anrechnung ersparter Aufrechnungen erhalten bleibt, müsse letztere Einschränkung auch in den durch $\$ 326$ Abs. 5 BGB in Bezug genommenen und im Übrigen funktionsgleichen $\$ 323$ Abs. 6 BGB hineingelesen werden. $\$ 326$ Abs. 2 S. 2 BGB könne damit bei »qualitativer Unmöglichkeit« im Falle eines Rücktrittausschlusses nach $\$ 323$ Abs. 6 BGB zumindest analog angewendet werden ${ }^{8}$.

\section{Stellungnahme}

Das vorstehend skizzierte Verständnis des Zusammenspiels von Kaufgewährleistungs- und allgemeinem Leistungsstörungsrecht vermag aus einer Reihe von Gründen nicht zu überzeugen.
1 LORENZ, NJW 2003, $1417 \mathrm{ff}$

2 Dauner-Lieb/Dötsch, ZGS 2003, $250 \mathrm{ff}$.

3 Vgl. nur die Replik von Lorenz, ZGS 2003, 398, 399 sowie die Duplik von DAUner-Lie в/Dö t SCH, ZGS 2003, $455 \mathrm{ff}$.

4 AG Daun NJW-RR 2003, 1465; AG Kempen MDR 2003, 1406 f. m. Anm. Dӧ т SCH. In beiden Entscheidungen wird ein solcher Anspruch abgelehnt; das AG Kempen verwirft in seiner Begründung zudem ausdrücklich die von Lo Renz vertretene Anwendung des $\$ 326$ Abs. 2 S. 2 BGB (dazu sogleich im Text).

5 Lorenz, NJW 2003, 1417, 1418, der selbst von »verfrühter Selbstvornahme« spricht.

6 Lorenz, NJW 2003, 1417, 1419; Ders., ZGS 2003, 398, 399; zustimmend nun Paland / Hein i ich s, BGB, 63. Aufl. 2004, \$326 Rdn. 9; vorsichtig in diese Richtung auch Münchener Kommentar-E R N S T, BGB, 4. Aufl. 2003, \$281 Rdn. 60. Hat der Käufer den Kaufpreis bereits gezahlt, so soll er einen entsprechenden Betrag gemäß $\$ \$ 326$ Abs. 4, 346 Abs. 1 BGB vom Verkäufer zurückfordern können.

7 Lorenz, NJW 2003, 1417, 1418; de rs., ZGS 2003, 398; ebenso Münchener Kommentar-Er N s T, BGB, 4. Aufl. 2003, \$281 Rdn. 60.

8 LORENZ, NJW 2003, 1417, 1419; so auch schon DERs., NJW 2002, 2497, 2499; nun auch Palandt/Heinrichs, BGB, 63. Aufl. 2004, \$326 Rdn. 13. 


\section{Vereitelung des "Rechts zur zweiten Andienung" des Verkäufers}

Zum einen würde durch die Zulassung eines Käuferrechts auf eigenmächtige Selbstvornahme das Recht des Verkäufers zur sog. "zweiten Andienung" ausgehebelt, welches nach der gesetzgeberischen Konzeption einen zentralen Bestandteil des neuen kaufrechtlichen Rechtsbehelfssystems darstellt ${ }^{9}$. Es hat im BGB freilich keine ausdrückliche eigenständige Normierung erfahren ${ }^{10}$, sondern ergibt sich gleichsam mittelbar daraus, dass der Käufer bei mangelhafter Lieferung ohne weitere Voraussetzung vom Verkäufer lediglich Nacherfüllung gemäß $\$ \$ 437$ Nr. 1, 439 Abs. 1 BGB verlangen kann, während alle sonstigen Rechtsbehelfe - Rücktritt und Minderung ( $\$ \$ 437$ Nr. 2, 440, 323, 326 Abs. 5, 441 BGB), Schadensersatz statt der Leistung und Ersatz vergeblicher Aufwendungen ( $\$ \$ 437$ Nr. 3, 440, 280, 281, 283, 311 a, 284 BGB) - im Regelfall zunächst die erfolglose Setzung einer Frist zur Nacherfüllung erfordern ${ }^{11}$. Die dadurch gesetzessystematisch gesicherte Möglichkeit des Verkäufers zur Nacherfüllung stellt sich im modernisierten Schuldrecht damit nicht lediglich als unbeabsichtigter Reflex des Fristsetzungserfordernisses, sondern als eigenes Recht des Verkäufers dar ${ }^{12}$.

Solange dieses Recht des Verkäufers zur Mängelbeseitigung besteht, ist der Käufer daher schon aus diesem Grund nicht berechtigt, den Mangel selbst zu beseitigen und die Kosten dem Verkäufer in Rechnung zu stellen ${ }^{13}$. Dies erscheint deshalb auch angemessen, weil es für den Verkäufer häufig wirtschaftlich vorteilhafter sein wird, die Nachbesserung in Natur vorzunehmen, als den Kostengegenwert in Geld zu zahlen ${ }^{14}$ (so mag er etwa über das zur Mängelbeseitigung notwendige Material und Personal verfügen, nicht hingegen über abrufbare Finanzmittel). Ein schützenswertes Recht des Käufers zur eigenmächtigen Selbstvornahme ist demgegenüber nicht ersichtlich; er ist vielmehr durch das ihm gemäß $\$ 439$ Abs. 1 BGB zustehende Wahlrecht zwischen der Beseitigung des Mangels und der Lieferung einer mangelfreien Sache durch den Verkäufer ${ }^{15}$, die aus $\$ 439$ Abs. 2 BGB folgende Belastung des Verkäufers mit den zum Zweck der Nacherfüllung erforderlichen Aufwendungen ${ }^{16}$ sowie die für verschiedene Konstellationen ( $\$ \$ 281$ Abs. 2, 323 Abs. 2, 326 Abs. 5, 440 BGB) angeordnete Entbehrlichkeit der Fristsetzung ausreichend geschützt. Durch das letztgenannte Regelungsinstrument wird ihm eine direkte Geltendmachung des Schadensanspruches etwa in den Fällen eröffnet, in denen der Verkäufer die Mängelbeseitigung verweigert oder das Vertrauensverhältnis zwischen den Parteien durch den Verkäufer so tiefgreifend gestört wurde, dass dem Käufer eine Verweisung auf den Nacherfüllungsanspruch schlechthin nicht zuzumuten ist ${ }^{17}$.

\section{Keine "Unmöglichkeit« der Mängelbeseitigungs- leistung nach Selbstvornahme}

Der hier kritisierte Ansatz, welcher die Folge der vom Käufer durchgeführten Selbstvornahme als einen Fall der Unmöglichkeit der Mängelbeseitigung i. S. d. $\$ 275$ Abs. 1 BGB einordnen ${ }^{18}$ und über diesen Weg sodann in Gestalt des $\$ 326$ Abs. 2 S. 2 BGB auf eine Norm des allgemeinen Leistungsstörungsrechts zurückgreifen will, überzeugt zudem aus einem weiteren Grunde nicht:

Zwar kann die Nacherfüllung durch den Verkäufer $(\$ 439$ Abs. 1 BGB) i. S.d. $\$ 275$ Abs. 1 BGB unmöglich sein, wenn nämlich ein unbehebbarer Mangel vorliegt. Um einen solchen Fall handelt es sich jedoch nicht, wenn der Käufer einen (ersichtlich behebbaren!) Mangel vorab selbst behoben hat. Ab diesem Zeitpunkt liegt nicht etwa nunmehr ein unbehebbarer Mangel ${ }^{19}$, sondern überhaupt kein Mangel mehr vor: Die Sache ist mangelfrei ${ }^{20} . \$ 275$ Abs. 1 BGB kann deshalb nicht eingreifen, weil diese Vorschrift einen »Anspruch auf Leistung « voraussetzt, den sie ab Unmöglichkeit (der Leistung) ausschließt: Ein Anspruch auf Mängelbeseitigung gemäß den $\$ \$ 437$ Nr. 1, 439 Abs. 1 BGB besteht aber nur dann, wenn die Sache zum Zeitpunkt des Nach- erfüllungsverlangens durch den Käufer noch mangelhaft ist. Dies ergibt sich daraus, dass sich der Käufer erst durch sein Nacherfüllungsverlangen zwischen Mängelbeseitigung und Ersatzlieferung entscheidet - ein rechtlicher Anspruch auf Mängelbeseitigung kann damit erst ab diesem Moment bestehen, nicht vorher $^{21}$. Eine Mängelbeseitigungsleistung kann folglich auch erst ab diesem Zeitpunkt geschuldet und ggfs. im Rechtssinne unmöglich sein. (Bei der alternativen Nacherfüllungsform der Ersatzlieferung - bei welcher in Selbstvornahmefällen also eine eigenmächtige Ersatzbeschaffung durch den Käufer vorläge - scheidet das Unmöglichkeitsargument dabei schon deshalb aus, weil dadurch die Behebbarkeit des (der ursprünglich gelieferten Sache weiterhin anhaftenden) Mangels in keiner Weise berührt wird ${ }^{22}$ : Auch an dieser Differenzierung, als solche eine zwingende Folge der hier kritisierten Argumentation, wird deren mangelnde Überzeugungskraft deutlich.)

In Konstellationen, in denen ein Sach- oder Rechtsmangel zwar bei Gefahrenübergang ( $\$ 434$ Abs. 1 BGB) vorliegt, aber zum Zeitpunkt des Nacherfüllungsverlangens nicht mehr besteht, scheidet ein Nacherfüllungsanspruch gemäß $\$ \$ 437$ Nr. 1, 439 Abs. 1 BGB daher aus. So gelagerte Fälle werden in der Praxis nicht eben häufig sein, sind aber durchaus denkbar: So mag die verkaufte Sache bei Gefahrenübergang mit dem Pfandrecht eines Dritten behaftet sein ( $\$ 435$ S. 1 BGB), der jedoch sodann von sich aus auf das Pfandrecht verzichtet; oder es wird - obgleich nach dem Kaufvertrag reife Früchte geschuldet sind - lediglich halbreife Ware übergeben, die jedoch bis zum Zeitpunkt des Nacherfüllungsverlangens nachgereift ist. Der Käufer bedarf in diesen Konstellationen eines Nacherfüllungsanspruches nicht: Er verfügt nunmehr ja über den Kaufgegenstand in der vereinbarten Beschaffenheit, und einen durch das verspätete Eintreten der

9 Die Begründung des Regierungsentwurfes, BT-Drs. 14/6040, S. 89 betont die »berechtigten Verkäuferinteressen«; so auch BÜDENBENDER, in: Dauner-Lieb/Heidel/Lepa/Ring, AnwaltKommentar, 2001, $\$ 437$ Rdn. 6: »entscheidende Neuausrichtung des Kaufrechts«; Palandt/Putzo, BGB, 63. Aufl. 2004, $\$ 437$ Rdn. 1.

10 Eine solche enthält hingegen das UN-Kaufrecht in Gestalt des Art. 48 CISG; vgl. H u в E R, in: Schlechtriem, CISG, 3. Aufl. 2000, Art. 48 Rdn. 1: "rechtspolitische Grundentscheidung ".

11 Lorenz, NJW 2002, 2497, 2499; SAEnger, in: Schulze/Dörner u. a. BGB-Handkommentar, 3. Aufl. 2003, 437 Rdn. 14.

12 AG Kempen MDR 2003, 1406; B iт t e R/Meid t, ZIP 2001, 2115, 2116; Boerner, ZIP 2001, 2264, 2269; Eн M A N N / S u t S CH e t, Modernisiertes Schuldrecht, 2002, S. 199; S A E N G E R, in: Schulze/Dörner u. a., BGBHandkommentar, 3. Aufl. 2003, \$439 Rdn. 1; S C H L E C H T R I E M, Schuldrecht Besonderer Teil, 6. Aufl. 2003, Rdn. 71; Tonner/EchtermeY E R, in: Kothe u. a., Das neue Schuldrecht: Kompaktkommentar, 2003, $\S 439$ Rdn. 18; a. A. We S T E R M A N N, JZ 2001, 530, 536.

13 AG Daun NJW-RR 2003, 1465; AG Kempen MDR 2003, 1406. Ebenso H u B E R, in: Schlechtriem, CISG, 3. Aufl. 2000, Art. 48 Rdn. 28 zur insoweit identischen Rechtslage nach dem UN-Kaufrecht.

14 In diesem Sinne SA E NG E R, in: Schulze/Dörner u. a., BGB-Handkommentar, 3. Aufl. 2003, $\$ 437$ Rdn. 14.

15 Zur dogmatischen Einordnung dieses Wahlrechts S P I C K H O F F, BB 2003, $589,590$.

16 AG Daun NJW-RR 2003, 1465.

17 Als Fälle der Unzumutbarkeit der Nacherfüllung werden die arglistige Täuschung durch den Verkäufer (Tonner/Crellwitz, in: Kothe u. a., Das neue Schuldrecht: Kompaktkommentar, 2003, \$440 Rdn. 16), Beeinträchtigungen der Vermögens- und Integritätsinteressen des Käufers aufgrund der Art, wie der Verkäufer seiner Pflicht nachkommt, sowie Beleidigungen anlässlich der Nachbesserung (B Ü DE N B E N DE R, in: Dauner-Lieb/Heidel/Lepa/Ring, AnwaltKommentar, 2001, \$440 Rdn. 9) genannt.

18 So auch Dauner-Lie B / Döt s Ch, ZGS 2003, 250.

19 So aber Lorenz, NJW 2003, 1417, 1418; insoweit skeptisch hingegen Dauner-Lie в / Dö t s CH, ZGS 2003, 250 Fn. 4; dies s, ZGS 2003, 455, 456.

20 Vorsichtig in diese Richtung auch DAU N E R-L I в в / D ö т s CH, ZGS 2003, $455,456$.

21 Vgl. Palandt/Heinrichs, BGB, 63. Aufl. 2004, \$437 Rdn. 10. 22 Dies gesteht auch Lorenz, NJW 2003, 1417, 1418 in Fn. 8 zu. 
Vertragsgemäßheit verursachten Schaden kann er über die $\$ \$ 437$ Nr. 3, 280 Abs. 2, 286 BGB geltend machen ${ }^{23}$. An einem Anspruch gemäß $\$ \$ 437$ Nr. 1, 439 Abs. 1 BGB, der durch die Selbstvornahme i. S. d. $\$ 275$ Abs. 1 BGB unmöglich werden und damit die in $\$ 326$ BGB normierten Folgen für den Gegenleistungsanspruch (auf Kaufpreiszahlung) auslösen könnte, fehlt es hingegen $^{24}$.

Hinzuzufügen ist, dass die zum alten Kaufgewährleistungsrecht kontrovers diskutierte Behandlung des späteren Wegfalls eines bei Gefahrenübergang bestehenden Mangels eine gänzlich andere rechtliche Problematik betraf ${ }^{25}$ : Man stritt, ob ein Wandelungs- oder Minderungsbegehren nach $\$ \$ 462,465$ BGB a. F. nur begründet sei, wenn der Mangel sowohl im Zeitpunkt des Gefahrenübergangs als auch bei Vollzug der Wandelung bzw. Minderung gegeben ist ${ }^{26}$, oder ob es allein auf den erstgenannten Zeitpunkt ankomme ${ }^{27}$. Für die hier interessierende Frage ist diese Diskussion unergiebig, hatte sie doch ihre Grundlage in einem anders ausgestalteten Rechtsbehelfssystem: Zum einen verhielt sie sich gar nicht zum Rechtsbehelf der Nacherfüllung, welcher im alten Recht nicht vorgesehen war und sich grundlegend von der funktionell dem heutigen Rücktritt entsprechenden Wandelung und der Minderung unterscheidet, und zum anderen entstand sie allein deshalb, weil Wandelung und Minderung im alten Recht keine einseitigen Gestaltungsrechte des Käufers waren, sondern zu ihrem Vollzug gemäß $§ 465$ BGB a. F. das Einverständnis des Verkäufers oder dessen Ersetzung durch eine gerichtliche Entscheidung voraussetzten ${ }^{28}$. Diese rechtstechnische Besonderheit bot wiederum erst den Anlass zur Frage, ob nach allgemeinen prozessualen Grundsätzen auf die Situation zum Zeitpunkt der mündlichen Verhandlung abzustellen sei (wodurch dem Verkäufer das Hinausgezögern seines Einverständnisses zum berechtigten Wandelungsbegehren des Käufers möglicherweise zugute kommen konnte) ${ }^{29}$. Im modernisierten Schuldrecht ist für diese Zweifel kein Raum mehr, da sämtliche Rechtsbehelfe des $\$ 437$ BGB nunmehr durch einseitige empfangsbedürftige Willenserklärung ausgeübt werden.

\section{3. §§ $437 \mathrm{ff}$. BGB als abschließende Regelung der Käuferrechtsbehelfe nach Gefahrenübergang}

Ein Recht des Käufers auf eigenmächtige Selbstvornahme der Mängelbeseitigung mit korrespondierender Kostenerstattung scheidet des weiteren deshalb aus, weil ein solches in der gesetzlichen Auflistung des $\$ 437$ BGB nicht enthalten ist, die ihrerseits eine abschließende Sonderregelung der Käuferrechtsbehelfe für die Zeit nach Gefahrenübergang darstellt ${ }^{30}$. Die häufig anzutreffende schlagwortartige Feststellung, seit der Schuldrechtsreform gebe es kein eigenständiges Gewährleistungsrecht mehr ${ }^{31}$, darf nicht darüber hinwegtäuschen, dass der Gesetzgeber die $\$ \$ 437 \mathrm{ff}$. BGB als besondere Regelung der Kaufgewährleistung für Sach- und Rechtsmängel ausgestaltet hat: Die im Ausgangspunkt für alle Schuldverhältnisse geltenden Vorschriften des allgemeinen Leistungsstörungsrechts kommen, sofern sie in den $\$ \$ 437 \mathrm{ff}$. BGB geregelte Sachfragen betreffen, ab dem Zeitpunkt des Gefahrenüberganges $^{32}$ eben nur noch zur Anwendung, soweit sie durch $\$ \$ 437 \mathrm{ff}$. BGB in Bezug genommen und damit rechtstechnisch zum Bestandteil des Kaufgewährleistungsrechts berufen wer$\operatorname{den}^{33}$. Für einen direkten Rückgriff auf die $\$ \$ 275 \mathrm{ff}$. BGB (und damit auch $\$ 326$ Abs. 2 S. 2 BGB, der durch die von $\$ 437$ BGB ausgehenden Verweisungsketten gerade nicht in Bezug genommen wird) ist hingegen kein Raum.

Die danach abschließende Aufzählung der Käuferrechte in $\$ 437$ BGB darf auch nicht durch eine Analogie zu anderen Normen unterlaufen werden, um auf diesem Wege das Rechtsbehelfssystem um ein Selbstvornahmerecht nebst Aufwendungsersatzanspruch zu »ergänzen«: Von einer planwidrigen Regelungslücke ${ }^{34}$ kann insoweit keine Rede $\operatorname{sein}^{35}$. Dies erschließt sich bereits aus einem Blick auf das Werkvertragsrecht, welches in
$\$ 637$ BGB ein Selbstvornahmerecht des Bestellers vorsieht; eine vergleichbare Norm wurde in das Kaufrecht - obgleich in der wissenschaftlichen Diskussion ausdrücklich angeregt ${ }^{36}$ - gerade nicht aufgenommen ${ }^{37}$. Auch in $\$ 637$ BGB werden das Recht zur Selbstvornahme und der Aufwendungsersatzanspruch im Übrigen von dem erfolglosen Ablauf einer Nachfrist zur Mängelbeseitigung abhängig gemacht; die eigenmächtige Selbstvornahme wäre danach selbst von dieser Norm nicht gedeckt ${ }^{38}$.

Gleichwohl ist es nicht so, als ob eine Befugnis des Käufers, die Beseitigung des Mangels nach erfolglosem Ablauf einer angemessenen Frist eigenständig vorzunehmen und hierfür Kostenersatz von dem Verkäufer zu verlangen, nach geltendem Recht nie bestünde $^{39}$ : Der Käufer kann dieses Resultat nämlich im Rahmen eines - nach Ablauf einer Frist gemäß $\$ 281$ Abs. 1 S. 1 BGB ja eröffneten - Schadensersatzverlangens nach $\$ \$ 437$ Nr. 3, 440, 280 Abs. 1, 3, 281 BGB herbeiführen. Die ihm entstandenen Kosten für die nach nicht erfolgter Nachbesserung durch den

23 Palandt/Putzo, BGB, 63. Aufl. 2004, \$437 Rdn. 36; Schlecht RIEM, Schuldrecht Besonderer Teil, 6. Aufl. 2003, Rdn.90. Für eine Anwendung des $\$ 280$ Abs. 1 BGB in diesem Fall hingegen mit beachtlichen Gründen Münchener Kommentar-Erns t, BGB, 4. Aufl. 2003, Vorbem. zu $\$ 275$ Rdn. 13. Der praktische Unterschied liegt in der Nichterforderlichkeit der von $\$ 286$ Abs. 1 BGB verlangten Mahnung.

24 Dass zeitlich nach dem in $\$ 434$ Abs. 1 BGB als relevant genannten Zeitpunkt des Gefahrenübergangs stattfindende Ereignisse Folgen für den Nacherfüllungsanspruch des Käufers zeitigen, liegt im Übrigen nur daran, dass dieser mit der Geltendmachung seines Nacherfüllungsverlangens abgewartet hat: Hätte er dies unmittelbar nach erfolgter Lieferung getan, wäre sein Anspruch unverändert geblieben.

25 Parallelen erkennen hingegen Dauner-Lieв/Dötsch, ZGS 2003, $455,456$.

26 So OLG Düsseldorf NJW-RR 1998, 265 sowie 1587, 1588; PALANDT/ P u t zo, BGB, 61. Aufl. 2002, \$ 462 Rdn. 9; offen gelassen von $B G H$ NJW 1996, 2647, 2648.

27 So BGH NJW 2001, 66, 67; OLG Ka R L S R U h e NJW-RR 1999, 279, 280.

28 Vgl. nur $B G H$ NJW 1996, 2647, 2648.

29 BGH NJW 2001, 66, 67.

30 AG Kempen MDR 2003, 1406; B Ü D E N B E N D E R, in: Dauner-Lieb/Heidel/ Lepa/Ring, AnwaltKommentar, 2001, $\$ 437$ Rdn. 1; OеткеR/ Mault Zs Ch, Vertragliche Schuldverhältnisse, 2002, S. 68; PAL ANDT/ Putzo, BGB, 63. Aufl. 2004, $\$ 437$ Rdn. 48; SaEnger, in: Schulze/ Dörner u. a., BGB-Handkommentar, 3. Aufl. 2003, $\$ 437$ Rdn. 19; T o N NER/ECH TER M E E R, in: Kothe u. a., Das neue Schuldrecht: Kompaktkommentar, 2003, $\$ 437$ Rdn. 1.

31 Statt aller Palandt/Heinrichs, BGB, 63. Aufl. 2004, Vorb v $\$ 275$ Rdn. 17.

32 Die wohl h. M. stellt insoweit auf den Gefahrenübergang ab (PA L A N D T / He in R I CH S, BGB, 63. Aufl. 2004, Vorb v $\$ 275$ Rdn. 17; S A E N Ge R, in: Schulze/Dörner u. a., BGB-Handkommentar, 3. Aufl. 2003, \$439 Rdn. 1), andere Stimmen hingegen auf den Zeitpunkt, in dem bei Mangelfreiheit der Kaufsache die Gegenleistungsgefahr auf den Käufer übergegangen wäre (H u в E R, NJW 2002, 1004, 1005) oder den Moment der »Lieferung«(OETKER/MAultzsch, Vertragliche Schuldverhältnisse, 2002, S. $69 \mathrm{ff}$.). Für die hier interessierenden Konstellationen führen alle Auffassungen zum selben Ergebnis.

33 Palandt/Heinrichs, BGB, 63. Aufl. 2004, Vorb v \$275 Rdn. 17; PA L A n t / Putzo, BGB, 63. Aufl. 2004, Überb v $\$ 433$ Rdn. 6.

34 So aber Lorenz, NJW 2003, 1417, 1418. Hingegen für eine direkte Anwendung des $\$ 326$ Abs. 2 S. 2 BGB nunmehr DE Rs., ZGS 2003, 398.

35 AG Kempen MDR 2003, 1406; B Ü D E N B E N DE R, in: Dauner-Lieb/Heidel/ Lepa/Ring, AnwaltKommentar, 2001, $\$ 437$ Rdn. 14; DAuner-Lie B/ Dö т sch, ZGS 2003, 250, 251; Dö т sch, MDR 2003, 1407.

36 So durch Tonner, VuR 2001, 87, 90.

37 BT-Drs. 14/6040, S. 229; DAU N E R-Li в в / D ӧ т S C H, ZGS 2003, 250, 251; in diesem Sinne auch LorEnz/RIEHM, Lehrbuch zum neuen Schuldrecht, 2002, Rdn. 634, 642.

38 Anzumerken ist zudem, dass wegen der bewusst unterschiedlichen Ausgestaltung von Kaufvertrags- und Werkvertragsrecht auch aus der Rechtsprechung zu $\$ 633$ BGB a. F. weder Argumente für noch gegen Ansprüche des Käufers im Falle der Selbstvornahme hergeleitet werden können (wie hier AG Kempen MDR 2003, 1406; Loren Z, ZGS 2003, 398, 399; a. A. DAU NeR-Li в / D Ö т s CH, ZGS 2003, 250, 251 f.; D i e s., ZGS 2003, 455, $457 \mathrm{f}$.).

39 So aber anscheinend BüDENBENDER, in: Dauner-Lieb/Heidel/Lepa/ Ring, AnwaltKommentar, 2001, §439 Rdn. 14. 
Verkäufer durchgeführte Selbstvornahme stellen dabei einen einfachen Schadensposten dar ${ }^{40}$. Der gesetzestechnische Unterschied zwischen dem so gegebenen Kostenerstattungsanspruch und dem Aufwendungsersatzanspruch bei Selbstvornahme des $\$ 637$ Abs. 1, 3 BGB besteht zum einen freilich darin, dass $\$ 280$ Abs. 1 S. 2 BGB (wenn auch vermutetes) Verschulden des Verkäufers voraussetzt; praktisch wird es allerdings nur selten vorkommen, dass der Verkäufer die Nichtvornahme der möglichen Nacherfüllung nicht zu vertreten hat ${ }^{41}$. Zum anderen richtet sich der Schadensersatzanspruch inhaltlich auf die Kompensation sämtlicher Kosten des mängelbeseitigenden Käufers, während ein Aufwendungsersatzanspruch nach $\$ 326$ Abs. 2 S. 2 BGB analog nur die (möglicherweise niedrigeren ${ }^{42}$ ) ersparten Aufwendungen des Verkäufers erfassen würde.

Eine sofortige Selbstvornahme ist hingegen nach dem geltenden Kaufrecht zudem deshalb unzulässig, weil dem Verkäufer durch eine vorzeitige Mängelbeseitigung mit Kostenpflicht möglicherweise das Recht genommen wird, die ihm aufgrund der Kosten unzumutbare Nachbesserung gemäß $\$ 439$ Abs. 3 BGB zugunsten einer Ersatzlieferung zu verweigern ${ }^{43}$.

Schließlich ist darauf hinzuweisen, dass man selbst bei einem direkten Rückgriff auf die $\$ \$ 323 \mathrm{ff}$. BGB - der, wie bereits erörtert, wegen des Charakters der $\$ \$ 437 \mathrm{ff}$. BGB als leges speciales unzulässig ist - nach richtiger Ansicht an einer Anwendung des $\$ 326$ Abs. 2 S. 2 BGB gehindert wäre: Hier ist nämlich vorrangig $\$ 326$ Abs. 1 S. 2 BGB zu beachten, der den Gegenleistungsanspruch, insoweit bewusst abweichend von den sonstigen Regelungen des allgemeinen Leistungsstörungsrechts, bei Unmöglichkeit der Nacherfüllungsleistung gerade ungeschmälert bestehen lässt. Zweck dieser Sondervorschrift ist wiederum die Vermeidung von Wertungswidersprüchen mit dem Kaufgewährleistungsrecht: Eine Reduktion des Kaufpreisanspruches soll nur möglich sein, soweit die in $\$ 437$ BGB genannten Rechtsbehelfe Minderung und Rücktritt - diese ermöglichen ${ }^{44}$. Da für den hier interessierenden Fall also auch $\$ 326$ Abs. 1 S. 2 BGB gezielt eine Ausnahme von den allgemeinen Unmöglichkeitsregeln anordnet, scheidet eine Umgehung dieser gesetzgeberischen Wertung auf dem Analogiewege auch aus diesem Grunde aus ${ }^{45}$.

\section{Zusammenfassung}

Es kann daher festgehalten werden, dass die Konstruktion eines Käuferrechts zur eigenmächtigen Selbstvornahme der Mängelbeseitigung nebst Kostenerstattungsanspruches auf der Grundlage des modernisierten Schuldrechts nicht in Frage kommt ${ }^{46}$.
Keiner weiteren Darlegung bedarf, dass auch ein Rekurs auf das Recht der Geschäftsführung ohne Auftrag oder die Regeln der ungerechtfertigten Bereicherung aufgrund des abschließenden Charakters des Kaufgewährleistungsrechts insoweit verschlossen ist ${ }^{47}$.

Darüber hinaus scheitert jedoch auch die vertragliche Vereinbarung eines sofortigen Selbstvornahmerechts in AGB, weil gemäß $\$ 309$ Nr. 4 BGB die $\$ \$ 281,323$ BGB, welche Gläubigerrechte von der Setzung einer Nachfrist abhängig machen, klauselfest sind ${ }^{48}$. Gleichermaßen unzulässig sind danach Klauseln, die zwar die Fristsetzung nicht ausdrücklich für entbehrlich erklären, deren Rechtsfolgen - etwa den auch Selbstvornahmekosten abdeckenden Schadensersatzanspruch - aber ipso facto eintreten lassen $^{49}$. Da das Nachfristerfordernis wegen $\$ \$ 305$ c, 307 Abs. 1 BGB zudem auch im Verkehr zwischen Unternehmen grundsätzlich unabdingbar ist ${ }^{50}$, steht mithin fest, dass ein Recht des Käufers zur eigenmächtigen Mängelbeseitigung mit nachfolgendem Kostenerstattungsanspruch auch auf diesem Wege nicht geschaffen werden kann.

40 Zutreffend Dauner-Lieв/Dötsch, ZGS 2003, 250, 251; Dötsch, MDR 2003, 1407; Schlechtriem, Schuldrecht Besonderer Teil, 6. Aufl. 2003, Rdn. 429.

41 Eнmann/Sutschet, Modernisiertes Schuldrecht, 2002, S. 258; so auch Dö т C H, MDR 2003, 1407, 1408.

42 So Lorenz, ZGS 2003, 398, 399; während DAuner-Lieb/Döt s CH, ZGS 2003, 455, 456 darauf hinweisen, dass sich beide Posten in der Praxis regelmäßig jedenfalls dann decken werden, wenn beide Parteien Dritte zur Reparatur einschalten müssen.

43 Die Vereitelung eines solchen Anspruchs auf Ersatzlieferung durch eigenmächtige Selbstvornahme führte nämlich konstruktiv nicht zu einem Anspruch nach $\$ 326$ Abs. 2 S. 2 BGB analog (dazu bereits oben sub. 2.).

44 BT-Drs. 14/6040, S. 189; Schlechtriem, Schuldrecht Allgemeiner Teil, 5. Aufl. 2003, Rdn. 439; Willingmann/Hirse, in: Kothe u.a., Das neue Schuldrecht: Kompaktkommentar, 2003, §326 Rdn. 6, 13.

45 A. A. Lorenz, NJW 2003, 1417, 1419.

46 AG Daun NJW-RR 2003, 1465; AG Kempen MDR 2003, 1406; B Ü DE NB E N DER, in: Dauner-Lieb/Heidel/Lepa/Ring, AnwaltKommentar, 2001, $\$ 437$ Rdn. 14; Daune R-Li в в/ Dö т s CH, ZGS 2003, 455, 458; ebenso Huber, in: Schlechtriem, CISG, 3. Aufl. 2000, Art. 48 Rdn. 28 zum Zusammenspiel von Art. 48 CISG mit $\$ 324$ Abs. 1 S. 2 BGB a. F.

47 Lorenz, NJW 2003, 1417，1419; Ders., ZGS 2003，398，399; Palandt/Putzo, BGB, 63. Aufl. 2004, $\$ 437$ Rdn. 57 f.; anders anscheinend Dö т S CH, MDR 2003, 1407, 1408.

48 Palandt/HeinRichs, BGB, 63. Aufl. 2004, \$309 Rdn. 22.

49 BGHZ 102, 41, 45.

50 BGHZ 110, 88, 97 